\title{
Systemic actinomycosis mimicking pelvic malignancy with pulmonary metastases
}

\author{
Edward Lawson MD FRCPC
}

E Lawson. Systemic actinomycosis mimicking pelvic malignancy with pulmonary metastases. Can Respir J 2005;12(3):153-154.

\begin{abstract}
A 40-year-old woman presented with profound weight loss, anemia and multiple pulmonary nodules. Further investigation revealed a hepatic abscess and a pelvic mass associated with an intrauterine device. Cultures of the hepatic abscess and from peripheral blood demonstrated Actinomyces species. The patient underwent an exploratory laparotomy and hysterectomy. Pathological examination showed characteristic sulphur granules of actinomycosis. Long-term therapy with high-dose intravenous penicillin resulted in clinical and radiographic improvement.
\end{abstract}

Key Words: Actinomycosis; Intrauterine device; Lung disease; Pelvic inflammatory disease

\section{Actinomycose systémique prenant les traits d'une néoplasie pelvienne avec métastases pulmonaires}

\begin{abstract}
Une femme de 40 ans a été admise pour perte de poids importante, anémie et nodules pulmonaires multiples. Des examens plus approfondis ont révélé un abcès hépatique et une masse au niveau pelvien associés à un dispositif intra-utérin. Des cultures de l'abcès hépatique et du sang périphérique ont confirmé la présence d'Actinomyces. La patiente a subi une laparotomie exploratoire et une hystérectomie. L'examen anatomopathologique a révélé la présence de granules de souffre caractéristiques de l'actinomycose. Un traitement à long terme par fortes doses de pénicilline intraveineuses a donné lieu à une amélioration, tant sur le plan clinique que radiographique.
\end{abstract}

A 40-year-old female smoker presented to the emergency room after coaxing by a worried husband and coworkers. She had experienced a $9.1 \mathrm{~kg}$ weight loss, anorexia and sweats over eight months. She denied any cough, dyspnea or chest pain. Amenorrhea had been present for several months but there was no abnormal discharge or pain. She complained of watery diarrhea and weakness of her nondominant hand.

The patient had a 30 pack-year smoking history. There was no history of respiratory infection, asthma, allergy or tuberculosis exposure. Her past medical and surgical history was unremarkable. No medications were currently prescribed and she did not engage in illicit drug use. She denied any behaviourial risk factors for HIV infection. There was no significant travel history or occupational exposure. She was married with one child and had an intrauterine device inserted several years previously. She worked full-time as a secretary. On systemic review, her only other complaint was of mouth irritation and pain. Her family history was noncontributory.

On physical examination, the patient appeared cachectic and anemic. Her vital signs were normal and oral thrush was present. Her cardiovascular and respiratory examinations were unremarkable. There was slight tenderness of the left lower quadrant with slight fullness. Pelvic and rectal examinations revealed a tender adnexal mass. There was no clubbing, rash or arthritis. Neurological examination revealed a left wrist drop with both motor and sensory loss in the radial nerve distribution.

Laboratory abnormalities demonstrated neutrophilia, severe microcytic anemia, hyponatremia, hypokalemia, and elevated C-reactive protein and ferritin. Renal function was normal and there were mild elevations in levels of liver enzymes. HIV testing

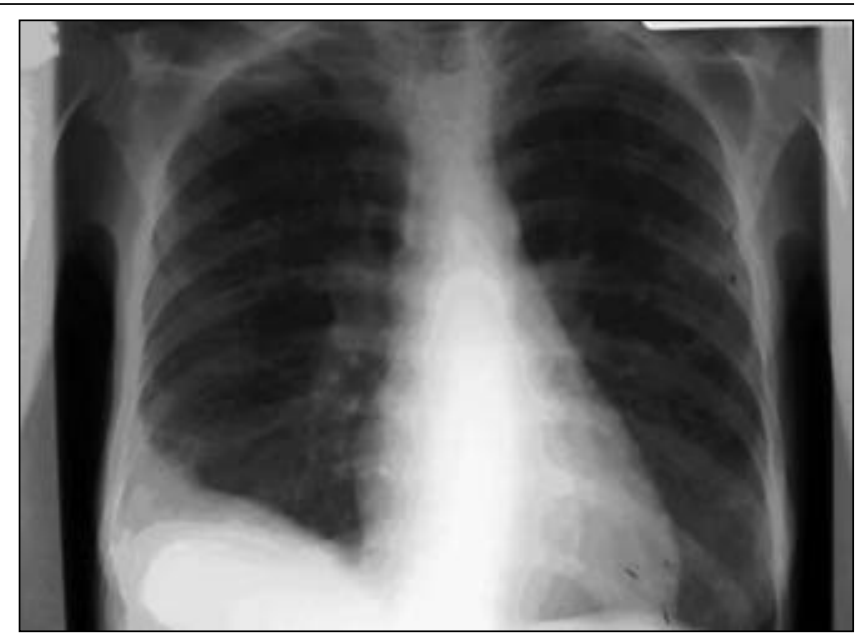

Figure 1) Plain chest radiograph demonstrating a right-sided pleural effusion and multiple pulmonary nodules of varying size

was negative. Serological tests for antinuclear antibody, angiotensin-converting enzyme and rheumatoid factor were negative. Complement levels were low. Antineutrophil cytoplasmic antibodies and proteinase 3 were weakly positive. Urinalysis showed 1+ protein, hematuria and many leukocytes. Urine culture yielded Escherichia coli and Klebsiella species. Blood cultures demonstrated Fusobacterium nucleatum and Gramnegative rods.

A plain chest radiograph (Figure 1) showed a right-sided pleural effusion and multiple small pulmonary nodules. Computed tomography of the chest (Figure 2) confirmed the presence of numerous 


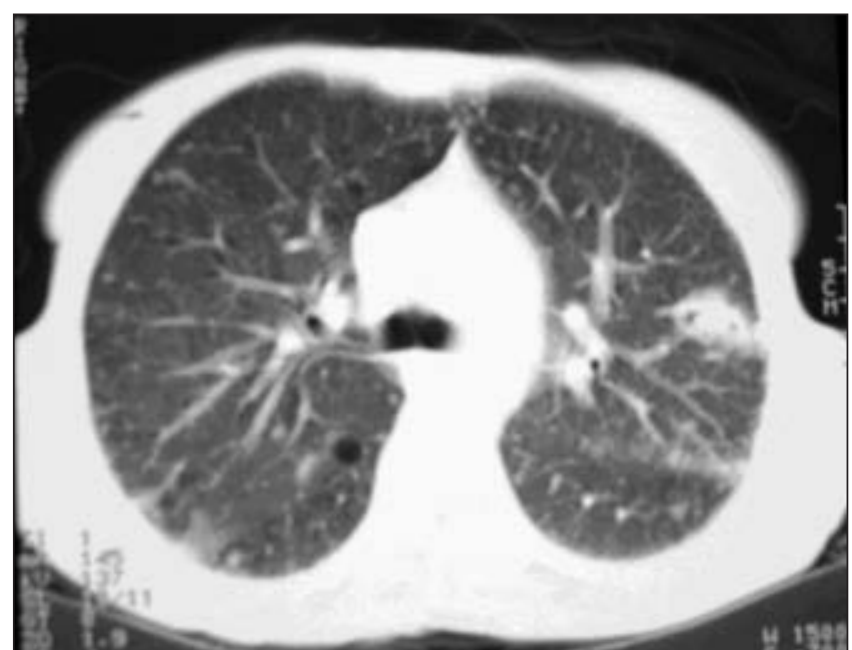

Figure 2) Chest computed tomography image showing multiple pulmonary nodules and a larger peripheral abscess.

small multilobar nodules ranging in size from several millimeters to $2 \mathrm{~cm}$ in diameter. There were small bilateral pleural effusions and a $3.0 \mathrm{~cm} \times 1.5 \mathrm{~cm}$ subcarinal lymph node. Ultrasound and computed tomography studies of the abdomen and pelvis revealed a low density hepatic mass, bilateral hydronephrosis, a $3.5 \mathrm{~cm}$ diameter right adnexal mass, a posteriorly thickened urinary bladder, periaortic and retrocaval lymphadenopathy, and mild splenomegaly. Colonoscopy revealed a perianal fistula and patchy ulceration of the rectum. Consultations from the gynecology, gastroenterology, general surgery and urology departments were obtained.

The patient underwent a liver biopsy that demonstrated pus and characteristic sulphur granules of Actinomyces species (Figure 3). Gram staining showed branching filamentous Grampositive bacteria, confirmed by culture as Actinomyces species. An abdominal hysterectomy and bilateral oophorectomy was performed revealing an infiltrated and chronically inflamed uterus with multiple tubo-ovarian abscesses. Penicillin $\mathrm{G}$ was administered intravenously for four weeks followed by a prolonged oral course. There was a dramatic clinical improvement, and follow-up thoracic imaging showed resolution in the nodular infiltrates. The patient had a complete recovery.

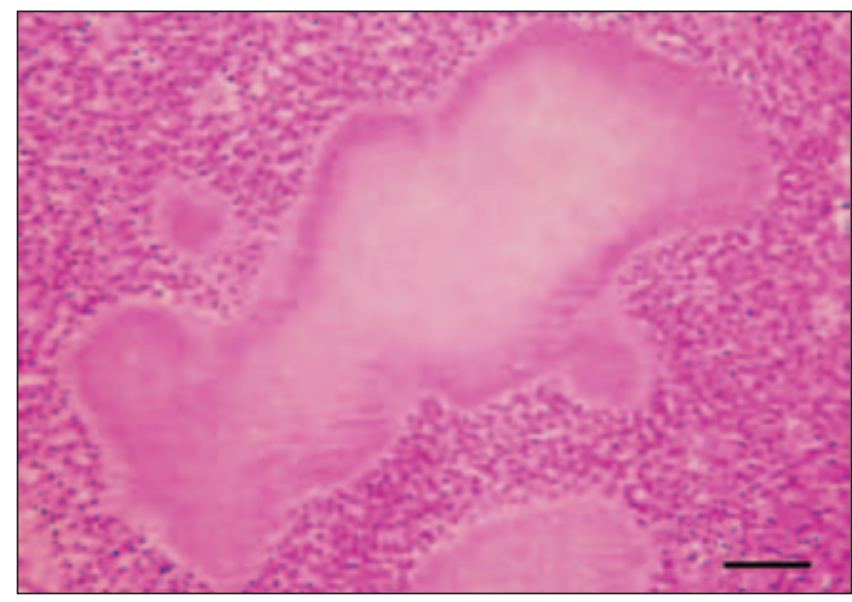

Figure 3) Liver biopsy specimen showing characteristic sulphur granules of actinomycosis infection (centre) in a neutrophil-rich inflammatory background (hematoxylin and eosin stain; scale bar $50 \mu \mathrm{m}$ )

\section{COMMENT}

Actinomycosis is an uncommon disease of the thorax, with most cases manifesting as chronic pleuropulmonary infections (1). Rarely, a systemic infection can occur with widespread metastatic foci. There are very few reports of systemic actinomycosis arising from underlying pelvic inflammatory disease $(2-4)$. The present case serves to remind that actinomycosis can present as an indolent progressive disease with systemic metastases (including pulmonary) that should be considered in the differential diagnosis of malignancy.

\section{REFERENCES}

1. Mabeza GF, Macfarlane J. Pulmonary actinomycosis. Eur Respir J 2003;21:545-51.

2. Lippes J. Pelvic actinomycosis: A review and preliminary look at prevalence. Am J Obstet Gynecol 1999;180:265-9.

3. Evans DT. Actinomyces israelli in the female genital tract: A review. Genitourin Med 1993;69:54-9.

4. Muller-Holzner E, Ruth NR, Abfalter E, et al. IUD-associated pelvic actinomycosis: A report of five cases. Int J Gynecol Pathol $1995 ; 14: 70-4$. 


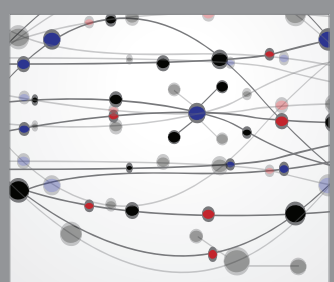

The Scientific World Journal
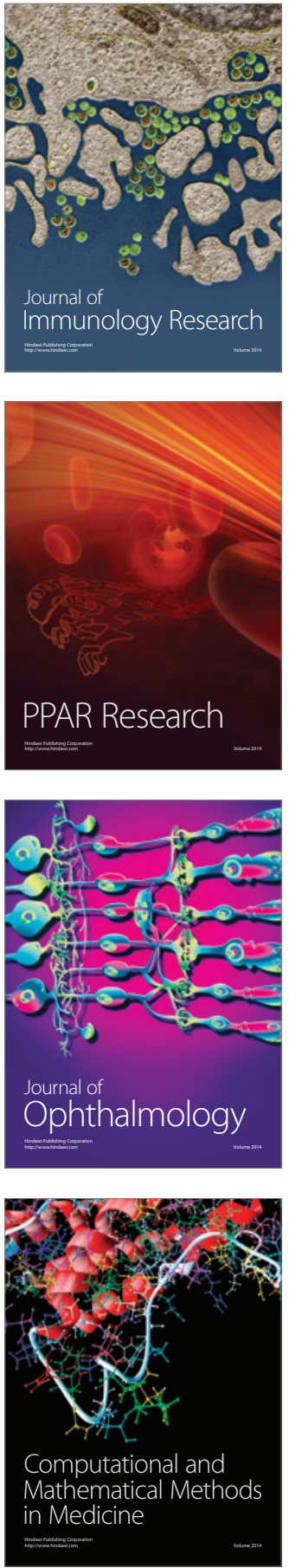

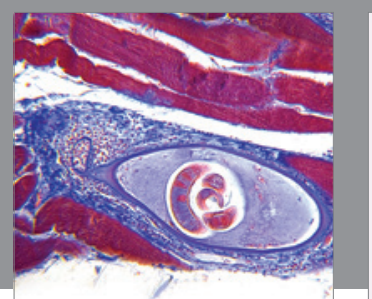

Gastroenterology Research and Practice

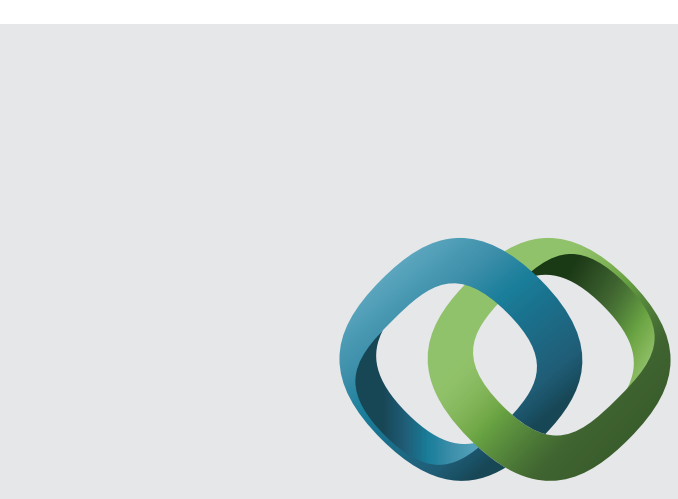

\section{Hindawi}

Submit your manuscripts at

http://www.hindawi.com
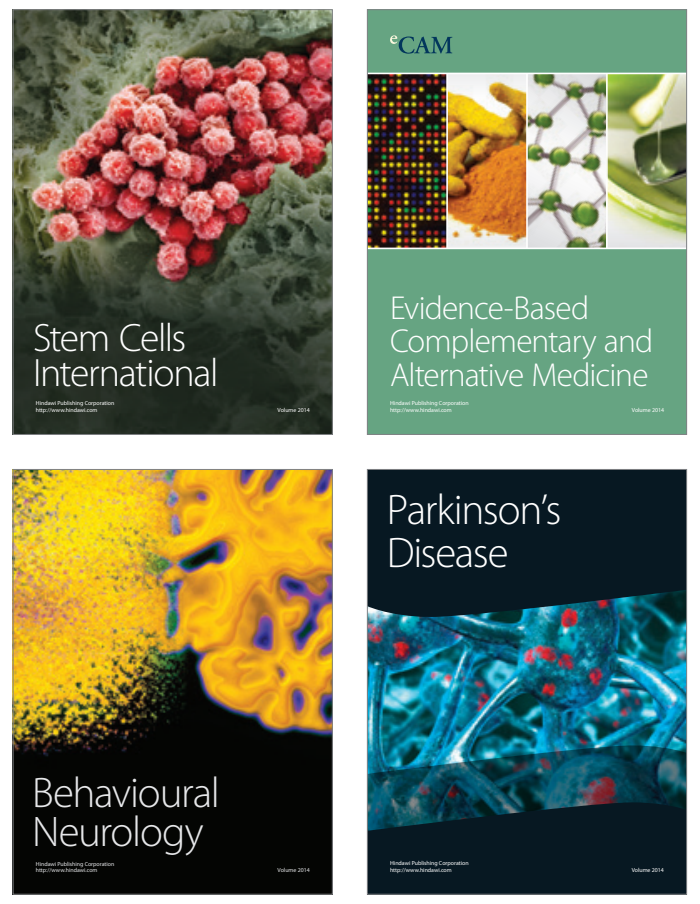
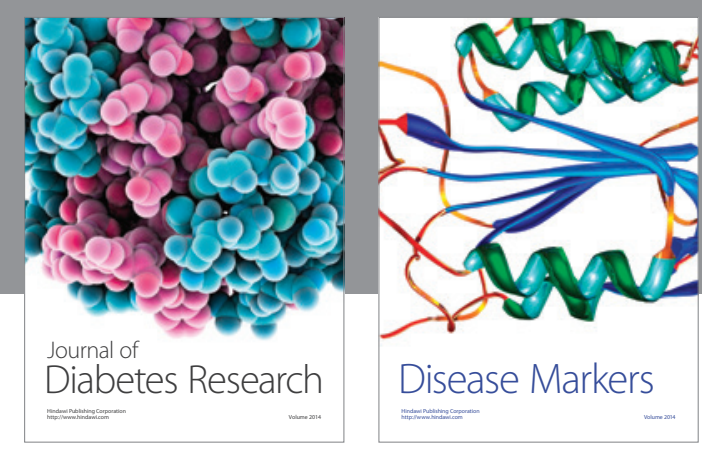

Disease Markers
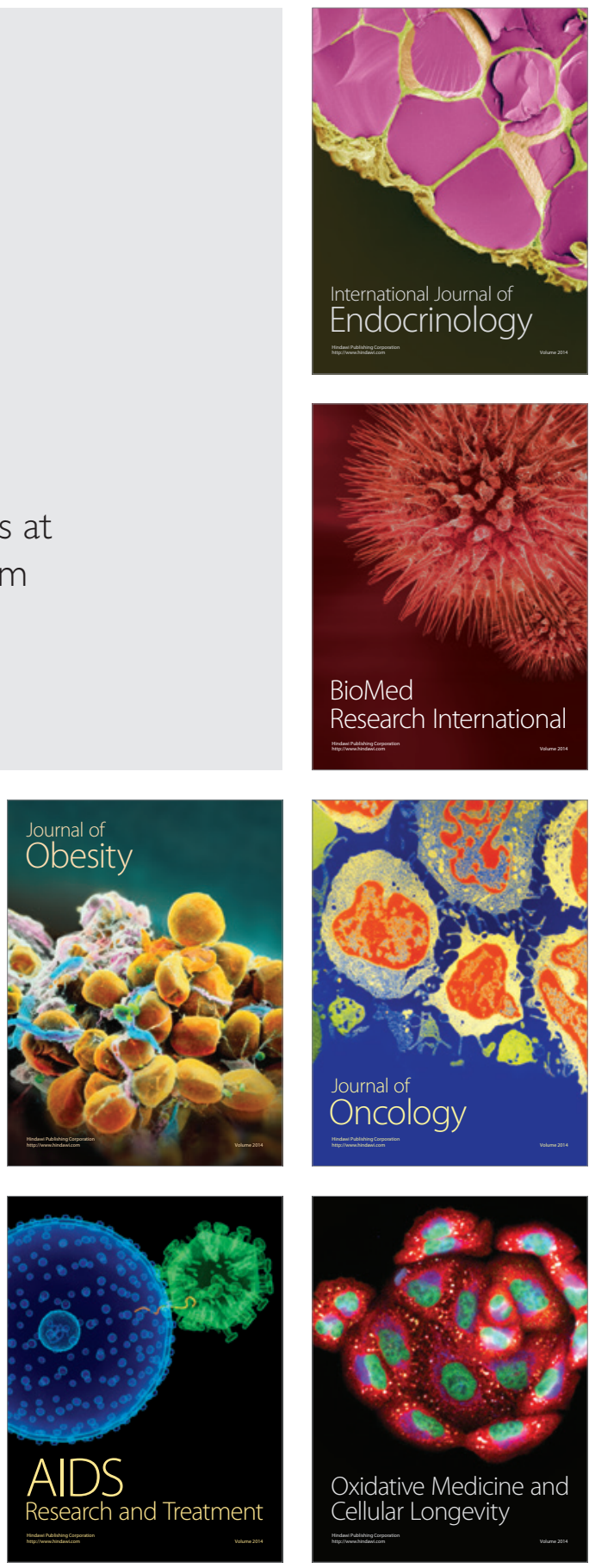